\title{
CORRELATION OF THE PATHOLOGICAL RESPONSE RATE WITH THE GLOBAL DISEASE AND SURVIVAL OF PATIENTS WITH SUB TYPE HER2 BREAST CANCER SUBMITTED TO NEOADJUVANT CHEMOTHERAPY AT HOSPITAL DE CÂNCER DE PERNAMBUCO
}

Juliana Beatriz de Oliveira Ferreira'1, Jose Peixoto1', Carolina de Souza Vasconcelos'1, Tainan de Morais Bispo' ${ }^{1}$ Hospital de Cancer de Pernambuco - Recife (PE), Brazil.

Introduction: QT NEO starts from the premise of tumor downstaging, enabling excision of previously unresectable tumors, reducing surgical extension and, consequently, enabling higher rates of conservative surgery. It also allows an in vivo assessment of the neoplastic response to systemic therapy. PCR is a powerful indicator of the benefit of QT NEO and is associated with better outcomes for relapse, DFS and OS. Objectives: This study aimed to evaluate the pathological response rate of patients with CM HER2 who underwent QT NEO, treated at HCP in the years 2014-2016 and to correlate with the recurrence rates, DFS and OS, observing whether the data are in agreement with those reported in the literature. Methods: The study evaluated patients with BC HER2, confirmed by IHC and/or FISH, who underwent QT NEO followed by surgery from 2014 to 2016, treated at the HCP. It is a retrospective, observational, and descriptive study. The information collected comes from a questionnaire formulated by the researcher with the relevant points to be analyzed in the project. The data were stored and analyzed using SPSS, version 20.0. The Kolmogorov-Smirnov test, $\chi^{2}$ test or Fisher's exact test were used to assess the variables. The DFS and OS time data were represented by the Kapplan-Meier curves and the Logrank test was used to verify a significant difference between the categories with or nPRC. A significance level of $5 \%$ was assigned to it. Results: The medical records of 58 patients with BC subtype HER 2 who received QT NEO followed by surgery were analyzed. During a mean follow-up of 35 months, 5 (8.6\%) local recurrences and 20 (34.5\%) distant ones and 18 deaths from breast cancer (31\%) were observed. A result of 50\% of PRC was achieved, with no correlation of statistical significance between age, histological type, nuclear grade, staging prior to QT NEO and chemotherapy regimen used. The PRC group achieved lower recurrence rates (20.68 vs. 51.72\%) with statistical significance. DFS in the PRC group is $75.9 \%$ and in the $\mathrm{nPRC}$ group it is $44.82 \%$. OS of the group with PRC is $82.7 v s .48 .2 \%$ in the nPRC group. Patients with HR- and PRC were the ones that most benefited from obtaining PRC in the recurrence and DFS outcomes, reaching recurrence rates of $22 \%$ and DFS of $77.8 \%$ compared to the HR group - without PRC, which had recurrence rates of $72 \%$ and DFS of 27.3\%. Conclusion: This study found significant data with lower recurrence rates and better rates of DFS and OS in patients who achieved PRC, thus evidencing the impact that PRC has on long-term outcomes, especially in the HRsubgroup of patients. 